\title{
Relation between giant magnetoresistance and critical current for spin precession in magnetic multilayers
}

\author{
L. Berger \\ Physics Department, Carnegie Mellon University, Pittsburgh, Pennsylvania 15213, USA
}

(Received 20 April 2005; published 26 September 2005)

\begin{abstract}
In a spin valve with current perpendicular to layers, the electrical resistance with antiparallel magnetic layers differs from the value with parallel layers, by an amount $\Delta R$. Also, above a certain critical value $I_{c}$, a dc current induces a precession of the magnetization in the free magnetic layer, through $s-d$ exchange. Using a local mechanism where current-induced torques depend only on the spin polarization of the current, we show that the average of $I_{c}$ over parallel and antiparallel states is inversely proportional to $\Delta R$ when almost any parameter of the spin valve is varied. This prediction agrees with the experimental results of Urazhdin et al. and of Emley et al. On the other hand, the kind of relation predicted in the case of the nonlocal mechanism, where currentinduced torques are mediated by the spin accumulation of Johnson and Silsbee, is inconsistent with some of the Urazhdin experiments. This shows that the local mechanism is dominant over the nonlocal one in these experiments.
\end{abstract}

DOI: 10.1103/PhysRevB.72.100402

PACS number(s): 73.50.Jt, 73.61.--r, 75.47.De

\section{INTRODUCTION}

We consider a spin valve [Fig. 1(a)] consisting of a pinned magnetic layer $F_{1}$, a spacer $N$, and a free magnetic layer $F_{2}$. The current leads $L_{1}$ and $L_{2}$ often have the shape of thick and flat electrodes. Sometimes, $F_{1}$ is left unpatterned so that it is part of the left-hand side electrode. A dc current $I$ runs in the direction normal to layers. The positive sense of $I$ is from the pinned to the free layer.

It is found ${ }^{1}$ that the parallel $(P)$ and antiparallel (AP) states of the spin valve, characterized by parallel and antiparallel magnetizations $\mathbf{M}_{1}, \mathbf{M}_{2}$ of layers $F_{1}, F_{2}$, respectively, [Fig. 1(a)] have different resistances $R_{P}, R_{A P}$, with $\Delta R$ $=R_{A P}-R_{P}>0$. The existence of $\Delta R$ is called giant magnetoresistance.

It has been predicted ${ }^{2,3}$ that the dc current $I$ would induce torques on $M_{2}$, through the $s$ - $d$ exchange interaction. Above a certain critical current $I_{c}$, these torques induce a precession of $\mathbf{M}_{2}$. This precession has been observed ${ }^{4}$ by several experimentalists.

A few theoretical authors ${ }^{5}$ have discussed $\Delta R$ and currentinduced torques in the same paper, but without stating or deriving any specific relation between the values of $\Delta R$ and $I_{c}$. On the other hand, Urazhdin et al. ${ }^{6}$ and Emley et al. ${ }^{7}$ have measured both $\Delta R$ and $I_{c}$ on the same series of samples, while varying certain characteristics of layers $F_{1}, N$, or $L_{2}$, and found $\Delta R$ and $I_{c}^{-1}$ to stay in a fixed ratio.

One purpose of the present paper is to derive a relation of proportionality between $\Delta R$ and $I_{c}^{-1}$, thus providing an explanation for the experimental findings of Urazhdin et al. ${ }^{6}$ and of Emley et al. ${ }^{7}$ A second purpose is to show that the relation is valid only for one of two possible physical mechanisms responsible for current-induced torques, thus suggesting which one of the two mechanisms is dominant in these experiments.

\section{EQUIVALENT CIRCUIT}

We show in Fig. 1(b) an equivalent electrical circuit for the spin valve of Fig. 1(a). The upper horizontal resistors represent conduction processes in the spin-down electron band, and the lower ones in the spin-up band. Even spin relaxation processes can be represented ${ }^{8}$ by an array of vertical resistors.

In the case of current leads of constant cross-section equal to that of the spin valve itself, it is found ${ }^{9}$ by solving socalled spin-diffusion equations that the infinite array of horizontal and vertical resistors representing the lead $L_{2}$ [Fig. 1(b) ] can be replaced by a simpler set of two horizontal resistors (Fig. 2) connected at one end, of values $R_{L 2}^{\uparrow}$ $=l_{L 2}^{s r} \rho_{L 2}^{\uparrow} / A, R_{L 2}^{\downarrow}=l_{L 2}^{s r} \rho_{L 2}^{\downarrow} / A$, where $l_{L 2}^{s r}, \rho_{L 2}^{\uparrow}, \rho_{L 2}^{\downarrow}$ and $A$ are the spin-diffusion length, spin-up and spin-down resistivities, and cross-section area of the lead. The reason why this substitution is permissible is that, at a distance $l_{L 2}^{s r}$ inside lead $L_{2}$, the spin-up and spin-down potentials become nearly equal so that the rest of the lead is unimportant.

As indicated by the formula above, the shorter the spinrelaxation time and the corresponding $l_{L 2}^{s r}$ value, the smaller

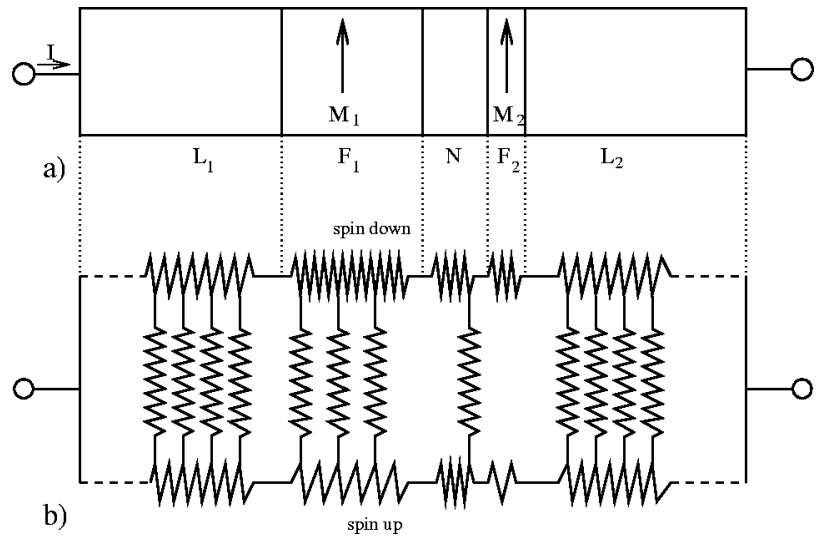

FIG. 1. (a) Spin valve where $F_{1}$ is the pinned magnetic layer and $F_{2}$ the free magnetic layer. (b) Equivalent electrical circuit for the spin valve under (a). The upper horizontal resistors represent conduction in the spin-down band, and the lower ones in the spin-up band. Vertical resistors describe the spin-relaxation processes. 


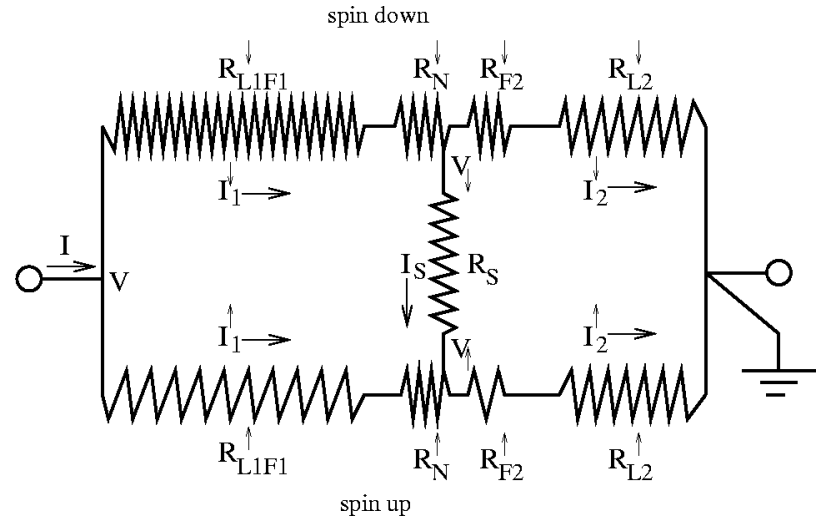

FIG. 2. Simplified equivalent circuit, where the infinite array of horizontal and vertical resistors representing lead $L_{2}$ [Fig. 1(b)] has been replaced by two horizontal resistors $R_{L 2}^{\uparrow}, R_{L 2}^{\downarrow}$. Also, the infinite array representing lead $L_{1}$ and unpatterned layer $F_{1}$ has been replaced by two horizontal resistors of effective values $R_{L 1 F 1}^{\uparrow}, R_{L 1 F 1}^{\downarrow}$.

$R_{L 2}^{\uparrow}$ and $R_{L 2}^{\downarrow}$ representing lead $L_{2}$. The effect of a thicker lead in the form of a flat electrode is ${ }^{10}$ to increase the crosssection area $A$, thus reducing $R_{L 2}^{\uparrow}$ and $R_{L 2}^{\downarrow}$ further and creating a partial "short circuit" between the spin-up and spindown bands. Similar considerations apply to lead $L_{1}$. If pinned magnetic layer $F_{1}$ is unpatterned like $L_{1}$, it is best lumped with it, with combined effective resistances called $R_{L 1 F 1}^{\uparrow}$ and $R_{L 1 F 1}^{\downarrow}$ (Fig. 2). On the other hand, we assume layers $N, F_{2}$ of resistances $R_{N}^{\uparrow}, R_{N}^{\downarrow}, R_{F 2}^{\uparrow}, R_{F 2}^{\downarrow}$ to be much thinner than a local spin-diffusion length, so that spin relaxation can be neglected in these layers. One exception will be the case where a fast relaxing material is incorporated into spacer $N$, represented by vertical resistor $R_{s}$ in Fig. 2 .

For simplicity, we assume the right-hand end of the circuit to be grounded (Fig. 2). The spin-up and spin-down electrochemical potentials in $F_{2}$ at the $N / F_{2}$ interface are called $V_{\uparrow}$ and $V_{\downarrow}$, while those at the left-hand end of the circuit are equal and called $V$. The spin-up and spin-down resistances of magnetic layers $F_{1}$ and $F_{2}$ include the resistance of adjacent interfaces. The $P$ state is the state shown in Fig. 2. In the AP state, $R_{F 2}^{\uparrow}$ and $R_{F 2}^{\downarrow}$ are exchanged. The spin-up and spin-down currents in $F_{1}$ are $I_{1}^{\uparrow}, I_{1}^{\downarrow}$ and those in $F_{2}$ are $I_{2}^{\uparrow}, I_{2}^{\downarrow}$. The spinflip current between the spin-up and spin-down bands through $R_{s}$ is $I_{s}$.

The Kirchoff equations for this circuit are

$$
\begin{gathered}
V-V_{\downarrow}=\left(R_{L 1 F 1}^{\downarrow}+R_{N}^{\downarrow}\right) I_{1}^{\downarrow} ; \quad V-V_{\uparrow}=\left(R_{L 1 F 1}^{\uparrow}+R_{N}^{\uparrow}\right) I_{1}^{\uparrow} ; \\
V_{\downarrow}=\left(R_{L 2}^{\downarrow}+R_{F 2}^{\downarrow}\right) I_{2}^{\downarrow} ; \quad V_{\uparrow}=\left(R_{L 2}^{\uparrow}+R_{F 2}^{\uparrow}\right) I_{2}^{\uparrow} ; \quad V_{\downarrow}-V_{\uparrow}=R_{s} I_{s} ; \\
I=I_{1}^{\downarrow}+I_{1}^{\uparrow} ; \quad I_{1}^{\downarrow}=I_{2}^{\downarrow}+I_{s} ; I_{1}^{\uparrow}=I_{2}^{\uparrow}-I_{s} .
\end{gathered}
$$

Assuming $R_{N}^{\uparrow}=R_{N}^{\downarrow}=2 R_{N}, R_{L 2}^{\uparrow}=R_{L 2}^{\downarrow}=2 R_{L 2}$, where $R_{N}, R_{L 2}$ are the resistances of $N$ and $L_{2}$, we solve Eqs. (1), and find the quantity $\Delta I=I_{2}^{\uparrow}-I_{2}^{\downarrow}$ related to the current polarization. The values of $\Delta I$ for the $P$ and AP states, called $\Delta I_{P}$ and $\Delta I_{A P}$, are different. Their average is found to be

$$
\frac{\Delta I_{P}+\Delta I_{A P}}{2}=I \frac{R_{L 1 F 1}^{\downarrow}-R_{L 1 F 1}^{\uparrow}}{\left(1+\frac{4 R_{L 2}}{R_{S}}\right)\left(R_{L 1 F 1}^{\uparrow}+R_{L 1 F 1}^{\downarrow}+4 R_{N}\right)+4 R_{L 2}} .
$$

The total spin-valve resistance is $R=V / I$, with values $R_{P}$ and $R_{A P}$ for the two states. With the same assumptions as before, we obtain from Eqs. (1) for $\Delta R=R_{A P}-R_{P}$,

$$
\Delta R=\frac{\left(R_{L 1 F 1}^{\downarrow}-R_{L 1 F 1}^{\uparrow}\right)\left(R_{F 2}^{\downarrow}-R_{F 2}^{\uparrow}\right)}{\left(1+\frac{4 R_{L 2}}{R_{S}}\right)\left(R_{L 1 F 1}^{\uparrow}+R_{L 1 F 1}^{\downarrow}+4 R_{N}\right)+4 R_{L 2}} .
$$

Finally, we obtain the spin accumulation $\overline{\Delta \mu}$ $=-e\left(V_{\uparrow}-V_{\downarrow}\right)$ in $F_{2}$ near the $N / F_{2}$ interface. This quantity has the dimension of an energy, and can be calculated from the equivalent circuit of Fig. 2 and Eqs. (1), with the same assumptions as before. Again, the $\overline{\Delta \mu}$ values in the $P$ and AP states, called $\overline{\Delta \mu}_{P}$ and $\overline{\Delta \mu}_{A P}$, are different, and their average is found to be

$$
\frac{\overline{\Delta \mu}_{P}+\overline{\Delta \mu}_{A P}}{2}=-\frac{\left(R_{2}^{\uparrow}+R_{2}^{\downarrow}+4 R_{2}^{L}\right)\left(R_{L 1 F 1}^{\downarrow}-R_{L 1 F 1}^{\uparrow}\right)}{\left(1+\frac{4 R_{L 2}}{R_{s}}\right)\left(R_{L 1 F 1}^{\uparrow}+R_{L 1 F 1}^{\downarrow}+4 R_{N}\right)+4 R_{L 2}} I e / 2
$$

Note that the quantities $\overline{\Delta \mu}_{P}, \overline{\Delta \mu}_{A P}, \Delta I_{P}, \Delta I_{A P}$ defined above are scalar rather than vectorial in nature; this is acceptable because we restrict ourselves to current values not exceeding the critical current for spin precession, where the layer magnetizations $\mathbf{M}_{1}, \mathbf{M}_{2}$ are still parallel or antiparallel. Conduction-electron spins precess rapidly ${ }^{2,3}$ around the $s-d$ exchange field $\simeq 100$ Tesla parallel to the magnetization, and electrons moving in different directions have uncorrelated precession phases. Thus, it is justified to average over all possible phases, giving a zero average for any transverse spin component. Only the longitudinal component of the spin ac- cumulation exists, below the critical current. Indeed, we found in the 1996 paper of Ref. 3, below Eq. (23), that the critical current can be expressed in terms of this scalar spin accumulation alone.

\section{LOCAL MECHANISM OF SPIN TRANSFER}

In the original papers on current-induced torques, ${ }^{2,3}$ translations of the spin-up and spin-down Fermi surfaces, and the resulting spin-polarized current, act directly and locally on layer $F_{2}$ to produce the torques. Thus, the critical currents 


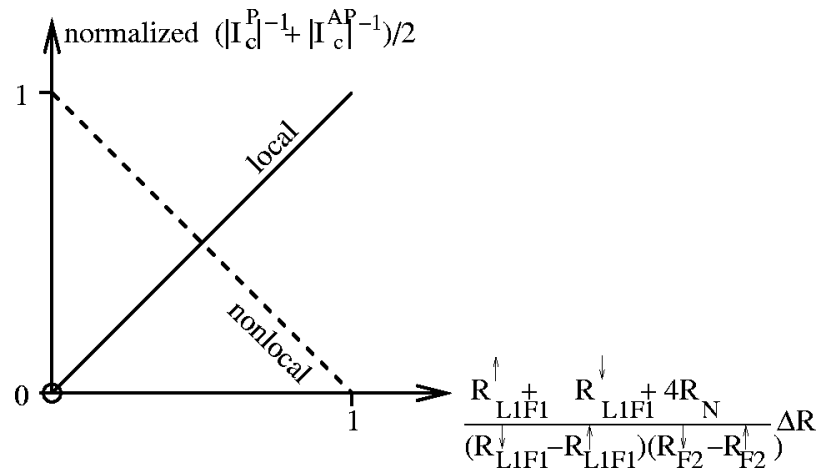

FIG. 3. Predicted relation between normalized values of $\left(\left|I_{c}^{P}\right|^{-1}\right.$ $\left.+\left|I_{c}^{A P}\right|^{-1}\right) / 2$ and $\Delta R$. The normalized $\Delta R$ value plotted horizontally is always $\quad \Delta R\left(R_{L 1 F 1}^{\uparrow}+R_{L 1 F 1}^{\downarrow}+4 R_{N}\right) /\left(R_{L 1 F 1}^{\downarrow}-R_{L 1 F 1}^{\uparrow}\right)\left(R_{F 2}^{\downarrow}-R_{F 2}^{\uparrow}\right)$. A solid line shows the prediction of Eq. (7) for the local mechanism, where the normalized value of $\left(\left|I_{c}^{P}\right|^{-1}+\left|I_{c}^{A P}\right|^{-1}\right) / 2$ plotted vertically is $\left.\quad\left(\left|I_{c}^{P}\right|^{-1}+\left|I_{c}^{A P}\right|^{-1}\right) / 2\right)\left(R_{L 1 F 1}^{\uparrow}+R_{L 1 F 1}^{\downarrow}+4 R_{N}\right) / C\left(R_{L 1 F 1}^{\downarrow}-R_{L 1 F 1}^{\uparrow}\right)$. Labels $P$ and $A P$ refer to the parallel and antiparallel states of the spin valve. A dashed line shows the prediction of Eq. (9) for the nonlocal mechanism, where $\left(\left|I_{c}^{P}\right|^{-1}+\left|I_{c}^{A P}\right|^{-1}\right) / \bar{C}\left(R_{L 1 F 1}^{\downarrow}-R_{L 1 F 1}^{\uparrow}\right) \quad$ is plotted vertically.

$I_{c}^{P}, I_{c}^{A P}$ for spin precession in the $P$ and AP states depend only on the corresponding spin polarizations $\Delta I_{P} / I, \Delta I_{A P} / I$ in $F_{2}$,

$$
\left(I_{c}^{P}\right)^{-1}=C \Delta I_{P} / I ; \quad\left(I_{c}^{A P}\right)^{-1}=-C \Delta I_{A P} / I .
$$

Here, $C$ is a positive constant depending only on the properties of layer $F_{2}$. If $F_{1}$ and $F_{2}$ are made of $\mathrm{Ni}$ or Co or their alloys, with $\Delta I_{P}, \Delta I_{A P}>0$, we have $I_{c}^{P}>0$ and $I_{c}^{A P}<0$. Combining Eqs. (2), (3), and (5), we obtain

$$
\frac{\left(\left|I_{c}^{P}\right|^{-1}+\left|I_{c}^{A P}\right|^{-1}\right) / 2}{\Delta R}=\frac{C}{R_{F 2}^{\downarrow}-R_{F 2}^{\uparrow}} .
$$

Note that $R_{L 1 F 1}^{\uparrow}, R_{L 1 F 1}^{\downarrow}, R_{s}, R_{N}$, and $R_{L 2}$ all have dropped out of this very simple relation. However, the relation between the individual $\left|I_{c}^{P}\right|$ or $\left|I_{c}^{A P}\right|$ and $\Delta R$ is much more complicated. Only in the limit $R_{F 2}^{\uparrow}, R_{F 2}^{\downarrow} \ll R_{L 2}$, where $\left|I_{c}^{P}\right| \simeq\left|I_{c}^{A P}\right|$ holds, would it become simple. This limit is not realized if layer $L_{2}$ is left unpatterned.

The right-hand side of Eq. (6) depends only on layer $F_{2}$. If we normalize $\Delta R$ and $\left(\left|I_{c}^{P}\right|^{-1}+\left|I_{c}^{A P}\right|^{-1}\right) / 2$ by a certain factor, this can be written in dimensionless form

$$
\begin{gathered}
\frac{\left|I_{c}^{P}\right|^{-1}+\left|I_{c}^{A P}\right|^{-1}}{2} \frac{\left(R_{L 1 F 1}^{\uparrow}+R_{L 1 F 1}^{\downarrow}+4 R_{N}\right)}{C\left(R_{L 1 F 1}^{\downarrow}-R_{L 1 F 1}^{\uparrow}\right)} \\
=\frac{\Delta R\left(R_{L 1 F 1}^{\uparrow}+R_{L 1 F 1}^{\downarrow}+4 R_{N}\right)}{\left(R_{L 1 F 1}^{\downarrow}-R_{L 1 F 1}^{\uparrow}\right)\left(R_{F 2}^{\downarrow}-R_{F 2}^{\uparrow}\right)} .
\end{gathered}
$$

This proportionality between $\left(\left|I_{c}^{P}\right|^{-1}+\left|I_{c}^{A P}\right|^{-1}\right) / 2$ and $\Delta R$ is represented by a solid straight line through the origin in Fig. 3 , at $45^{\circ}$ to the horizontal axis. The relation is valid as long as the properties of $F_{2}$ are not changed.

Urazhdin et al. ${ }^{6}$ have measured $\Delta R$ as well as $I_{c}^{P}$ and $I_{c}^{A P}$ on a $\mathrm{Cu}(80 \mathrm{~nm}) / \mathrm{Ni}_{84} \mathrm{Fe}_{16}(30 \mathrm{~nm}) / \mathrm{Cu}(15 \mathrm{~nm}) / \mathrm{Ni}_{84} \mathrm{Fe}_{16}$ $\times(6 \mathrm{~nm}) / \mathrm{Cu}(2 \mathrm{~nm}) / \mathrm{Au}(150 \mathrm{~nm})$ spin valve. The $30-\mathrm{nm}-$ thick $\mathrm{Ni}-\mathrm{Fe}$ layer is the pinned layer $F_{1}$, and was left unpat- terned (see Sec. II). The 6-nm-thick $\mathrm{Ni}-\mathrm{Fe}$ layer is the free layer $F_{2}$. In one kind of experiment, they replaced the $\mathrm{Cu}(2 \mathrm{~nm})$ layer near the right-hand side lead by a fastrelaxing $\mathrm{Cu}(2 \mathrm{~nm}) / \mathrm{Fe}_{50} \mathrm{Mn}_{50} / \mathrm{Cu}(2 \mathrm{~nm})$ sandwich, thus reducing the effective spin-diffusion length and effective resistance $R_{L 2}$ of the lead (see Sec. II). In another series of experiments, they replaced part of the $\mathrm{Cu}(15 \mathrm{~nm})$ spacer by a fast-relaxing $\mathrm{Cu}_{94} \mathrm{Pt}_{6}$ layer of thickness $d=4$, 8 , or $12 \mathrm{~nm}$, thus reducing $R_{s}$ from infinity (Fig. 2). The $\left(\left|I_{c}^{P}\right|^{-1}\right.$ $\left.+\left|I_{c}^{A P}\right|^{-1}\right) / 2$ and $\Delta R$ values were in all cases approximately proportional to each other, in agreement with the predictions of Eqs. (6) and (7) and Fig. 3 for the local mechanism. The measured individual $\left|I_{c}^{P}\right|$ and $\left|I_{c}^{A P}\right|$ were rather close to each other but, for reasons indicated below Eq. (6), we cannot compare these individual values to our theory.

Emley et $a l^{7}$ have measured $\Delta R$ as well as $I_{c}^{P}$ and $I_{c}^{A P}$ on $\mathrm{Cu}(100 \mathrm{~nm}) / \mathrm{Co}(8 \mathrm{~nm}) / \mathrm{Cu}(6 \mathrm{~nm}) / \mathrm{Co}(2 \mathrm{~nm}) / \mathrm{Cu}(2 \mathrm{~nm}) / \mathrm{Pt}$ $\times(30 \mathrm{~nm})$ spin valves, as well as on ones where the $\mathrm{Co}(8 \mathrm{~nm})$ pinned layer, corresponding to $F_{1}$, is replaced by a $\mathrm{Co}(11.5 \mathrm{~nm}) / \mathrm{Ru}(0.7 \mathrm{~nm}) / \mathrm{Co}(8 \mathrm{~nm})$ synthetic antiferromagnet. The effect of that substitution was to decrease both $\left(\left|I_{c}^{P}\right|^{-1}+\left|I_{c}^{A P}\right|^{-1}\right) / 2$ and $\Delta R$ by a factor of approximately 2 . Again, we see that $\left(\left|I_{c}^{P}\right|^{-1}+\left|I_{c}^{A P}\right|^{-1}\right) / 2$ and $\Delta R$ are nearly proportional to each other, in agreement with Eqs. (6) and (7) for the local mechanism.

\section{NONLOCAL MECHANISM OF SPIN TRANSFER}

Apart from the local mechanism associated with Fermisurface translation (Sec. III), there is ${ }^{8}$ a second mechanism that is less local and more diffusive. The current-induced torques do not depend on $\Delta I$ as for the local mechaanism, but rather on the spin accumulation $\overline{\Delta \mu}$ of Johnson and Silsbee. ${ }^{11}$ That spin accumulation is connected ${ }^{8,9}$ with an isotropic expansion or contraction of the Fermi surface, and depends on the physical properties of the spin valve and of the current leads over distances equal to one spin-diffusion length. In his 2002 paper, ${ }^{12}$ Slonczewski seems to take into account both mechanisms. Stiles and Zangwill ${ }^{13}$ use the nonlocal mechanism only. The spin accumulation is of order $10^{-3} \mathrm{eV}$ at the critical current.

Since $\overline{\Delta \mu}$ now mediates the current-induced torques, we have instead of Eq. (6),

$$
\left(I_{c}^{P}\right)^{-1}=\bar{C} \frac{\overline{\Delta \mu}_{P}}{I e} ; \quad\left(I_{c}^{A P}\right)^{-1}=-\bar{C} \frac{\overline{\Delta \mu}_{A P}}{I e},
$$

where $\bar{C}$ is a constant that depends only on the properties of $F_{2}$.

By combining Eqs. (8), (4), and (3), we obtain instead of Eq. (6),

$$
\frac{\left(\left|I_{c}^{P}\right|^{-1}+\left|I_{c}^{A P}\right|^{-1}\right) / 2}{\Delta R}=\bar{C} \frac{R_{F 2}^{\uparrow}+R_{F 2}^{\downarrow}+4 R_{L 2}}{2\left(R_{F 2}^{\downarrow}-R_{F 2}^{\uparrow}\right)} .
$$

Again, only in the limit $R_{F 2}^{\uparrow}, R_{F 2}^{\downarrow} \ll R_{L 2}$, where $\overline{\Delta \mu}_{P}$ $\simeq \overline{\Delta \mu}_{A P}$ holds, does a simple relation exist between the individual critical currents and $\Delta R$. And that limit is not usually realized. 
Note that the right-hand side of this equation depends only on $F_{2}$ and $L_{2}$, so that the equation is useful whenever these layers are kept unchanged. The proportionality relation of Eq. (9) is similar to that of Eqs. (6) or (7) for the local mechanism. Therefore, those Urazhdin experiments where $R_{s}$ is varied, as well as the Emley experiments where the nature of $F_{1}$ is changed (see Sec. III), can be explained by either of the two mechanisms.

Comparing Eq. (9) to Eq. (6) suggests that only by varying $R_{L 2}$ can the two mechanisms be distinguished. Therefore, we should consider those Urazhdin experiments where the effective resistance $R_{L 2}$ of lead $L_{2}$ is varied by putting a fast-relaxing material near that lead (see Sec. III). Trying to explain them with the nonlocal model, we eliminate $R_{L 2}$ between Eqs. (3) and (4). Assuming $R_{s}=\infty$ and using Eq. (8), we obtain a relation between $\left(\left|I_{c}^{P}\right|^{-1}+\left|I_{c}^{A P}\right|\right) / 2$ and $\Delta R$ where $R_{L 2}$ does not appear anymore,

$$
\frac{\left(R_{L 1 F 1}^{\uparrow}+R_{L 1 F 1}^{\downarrow}+4 R_{N}\right)}{\left(R_{F 2}^{\downarrow}-R_{F 2}^{\uparrow}\right)\left(R_{L 1 F 1}^{\downarrow}-R_{L 1 F 1}^{\uparrow}\right)} \Delta R+\frac{\left|I_{c}^{P}\right|^{-1}+\left|I_{c}^{A P}\right|^{-1}}{\bar{C}\left(R_{L 1 F 1}^{\downarrow}-R_{L 1 F 1}^{\uparrow}\right)}=1 .
$$

The normalization factor for $\Delta R$ is the same as in Eq. (7), so that Eq. (10) can also be represented in Fig. 3. It appears as a dashed straight line at $45^{\circ}$ not passing through the origin. This line is very different from the solid line passing through the origin coming from Eq. (7) and the local mechanism of Sec. III; for example, $\Delta R$ and $\left(\left|I_{c}^{P}\right|^{-1}+\left|I_{c}^{A P}\right|^{-1}\right) / 2$ now vary in opposite directions. Moreover, this line is not consistent with the Urazhdin data above, which show a proportionality between $\left(\left|I_{c}^{P}\right|^{-1}+\left|I_{c}^{A P}\right|^{-1}\right) / 2$ and $\Delta R$. Therefore, these data can only be explained by the local mechanism.

\section{CONCLUSIONS AND FINAL REMARKS}

A dc current induces drive torques on the magnetization of a magnetic layer through two different mechanisms. ${ }^{8}$ One mechanism is more local and ballistic, and depends directly on the current polarization, while the other is more nonlocal and diffusive, and depends on the spin accumulation. We derive relations between the giant magnetoresistance $\Delta R$ and an average of the critical currents $I_{c}^{P}$ and $I_{c}^{A P}$ for spin precession, in the case of the local (Sec. III) as well as of the nonlocal mechanism (Sec. IV).

Urazhdin et al. ${ }^{6}$ and Emley et al. ${ }^{7}$ have measured $\Delta R$ as well as $I_{c}^{P}$ and $I_{c}^{A P}$ on a series of samples, and some of their results are consistent with both local and nonlocal mechanisms. But the results of Urazhdin et al. obtained while varying the spin-relaxation rate near one lead are consistent only with the local mechanism, suggesting that this mechanism is dominant in their samples.

This conclusion is not surprising. In Urazhdin's samples, the pinned layer $F_{1}$ is unpatterned, and is really part of the thick left-hand side lead $L_{1}$ as discussed in Sec. II. Since it is made of $\mathrm{Ni}-\mathrm{Fe}$ with a very short spin-diffusion length $l^{s r}$ $\simeq 6 \mathrm{~nm}$, the effective spin-up and spin-down resistances of that lead will be unusually small (see Sec. II). According to Ref. 10 , this tends to boost $\Delta I$ and decrease the magnitude of $\overline{\Delta \mu}$ in $F_{2}$, tending to make the local mechanism dominant. The importance of the current polarization, i.e., of $\Delta I$, was already noted by Urazhdin et al. ${ }^{6}$

In 2000, Gu et al. ${ }^{14}$ had already shown that the insertion of a fast-relaxing FeMn layer near one lead of a spin valve would increase $\Delta R$, in agreement with the local mechanism.

Nevertheless, it is possible ${ }^{10}$ to design spin valves where the nonlocal mechanism is dominant because $|\overline{\Delta \mu}|$ is large and $\Delta I$ small. This is done by keeping layers $F_{1}, N$, and $F_{2}$ far away from both thick leads, and avoiding spin-relaxing materials. Another case where the nonlocal mechanism is dominant is the so-called "antisymmetric" spin valve, ${ }^{15}$ with one extra pinned magnetic layer on the other side of the free layer. In that layer configuration, $|\overline{\Delta \mu}|$ reaches in $F_{2}$ values three times larger than usual, and is expected to be unaffected by the nature of the leads because $\overline{\Delta \mu} \simeq 0$ in these leads.
${ }^{1}$ J. Bass and W. P. Pratt, J. Magn. Magn. Mater. 200, 274 (1999).

${ }^{2}$ J. C. Slonczewski, J. Magn. Magn. Mater. 159, L1 (1996); 195, L261 (1999).

${ }^{3}$ L. Berger, Phys. Rev. B 54, 9353 (1996); L. Berger, J. Appl. Phys. 81, 4880 (1997); 91, 6795 (2002).

${ }^{4}$ M. Tsoi, A. G. M. Jansen, J. Bass, W. C. Chiang, M. Seck, V. Tsoi, and P. Wyder, Phys. Rev. Lett. 80, 4281 (1998); E. B. Myers, D. C. Ralph, J. A. Katine, R. N. Louie, and R. A. Buhrman, Science 285, 867 (1999); J. Z. Sun, J. Magn. Magn. Mater. 202, 157 (1999).

${ }^{5}$ A. A. Kovalev, A. Brataas, and G. E. W. Bauer, Phys. Rev. B 66, 224424 (2002); G. E. W. Bauer, Y. Tserkovnyak, D. HuertasHernando, and A. Brataas, ibid. 67, 094421 (2003).

${ }^{6}$ S. Urazhdin, N. Birge, W. P. Pratt, and J. Bass, Appl. Phys. Lett. 84, 1516 (2004).
${ }^{7}$ N. C. Emley, F. J. Albert, E. M. Ryan, I. N. Krivorotov, D. C. Ralph, R. A. Buhrman, J. M. Daughton, and A. Jander, Appl. Phys. Lett. 84, 4257 (2004).

${ }^{8}$ L. Berger, J. Appl. Phys. 89, 5521 (2001); A. Fert and S. F. Lee, Phys. Rev. B 53, 6554 (1996).

${ }^{9}$ L. Berger, IEEE Trans. Magn. 34, 3837 (1998). See Eq. (5).

${ }^{10}$ L. Berger, J. Magn. Magn. Mater. 278, 185 (2004).

${ }^{11}$ M. Johnson and R. H. Silsbee, Phys. Rev. Lett. 55, 1790 (1985).

${ }^{12}$ J. C. Slonczewski, J. Magn. Magn. Mater. 247, 324 (2002). See Sec. 8.

${ }^{13}$ M. D. Stiles and A. Zangwill, Phys. Rev. B 66, 014407 (2002). See Eqs. (41) and (42).

${ }^{14}$ J. Y. Gu, S. D. Steenwik, A. C. Reilly, W. Park, R. Loloe, J. Bass, and W. P. Pratt, J. Appl. Phys. 87, 4831 (2000).

${ }^{15}$ L. Berger, J. Appl. Phys. 93, 7693 (2003). 